\title{
Template-based Prediction of Protein 8-state Secondary Structures
}

\author{
Ashraf Yaseen \\ Department of Computer Science \\ Old Dominion University, Norfolk, VA \\ ayaseen@cs.odu.edu
}

\author{
Yaohang Li \\ Department of Computer Science \\ Old Dominion University, Norfolk, VA \\ yaohang@cs.odu.edu
}

\begin{abstract}
Accurately predicting protein secondary structures is important to many protein structure modeling applications. In this paper, we investigate a template-based approach to enhance 8-state secondary structure prediction accuracy. The rationale is to construct structural templates from known protein structures with certain sequence similarity. The information contained in templates is then incorporated as features with sequence, evolutionary, and heuristic information to train neural networks. Our computational results show that templates containing structural information are effective features to enhance 8-state secondary structure prediction. A 7-fold cross-validated Q8 score of $\mathbf{7 8 . 8 5 \%}$ is obtained.
\end{abstract}

Keywords- Protein Secondary Structures, Neural Networks, Homology Templates

\section{INTRODUCTION}

An important intermediate step in modeling the threedimensional structure of a protein is to accurately predict the secondary structures [1]. Most often, the secondary structures are classified into three general states, i.e., helices $(\mathrm{H})$, strands (E), and coils (C). Correspondingly, performance of secondary structure prediction is typically measured by the Q3 accuracy. Many machine learning methods, including neural networks, hidden Markov chain, support vector machines, have been developed to predict secondary structures. Correspondingly, there are many secondary structure prediction servers available, including PSI-Pred [2], PHD [3], Porter [4], JPred [5], SSPRO [6], NETSURF [7], and many others. The modern secondary structure prediction servers can generate prediction results of $\sim 80 \%$ Q3 accuracy.

Compared to the general three secondary structure states, the DSSP program [8] has more detailed classifications by assigning secondary structures to eight states, including 3-10 helix (G), $\alpha$-helix (H), $\pi$-helix (I), $\beta$-stand (E), bridge (B), turn $(\mathrm{T})$, bend $(\mathrm{S})$, and others $(\mathrm{C})$. The 8 -state secondary structures convey more precise structural information than 3state, which is particularly important for a variety of applications.

Most current secondary structure perdition methods, including those for 8-state predictions [6,9], do not rely on similarity to known protein structures. However, many protein sequences have some degree of similarity among themselves. Actually, over half of all known protein sequences have some detectable similarity $(>25 \%)$ to one or more sequences of known structures [10]. Consequently, taking advantage of structural similarity of proteins with sequence similarity may lead to significant improvement of protein structure prediction. In fact, the latest version of porter has used homology-based templates for 3-state secondary structure prediction [10]. Porter has been reported to achieve prediction accuracy improvement when known structures with $>30 \%$ sequence similarity are available and even reach theoretical upper bound when such sequence similarity is higher than $50 \%$.

In this paper, we investigate a template-based method for 8 -state secondary structure prediction. We extract structural information from known structures of chains with certain sequence similarity to build structural templates. Then, the structural template is incorporated as features together with sequence, evolutionary, and heuristic information for neural network training and validation. We test our prediction method on several popularly used benchmarks.

\section{MATERIALS AND METHODS}

\section{A. The Protein Data Sets}

We use the protein dataset Cull5547 from PISCES server [11] for neural network training. Cull5547 contains 5547 protein chains with at most $25 \%$ sequence identity and $2.0 \mathrm{~A}$ resolution cutoff. Public benchmarks, including CB513 [12], Manesh215 [13], Carugo338 [14], and CASP9 targets [15] are used to benchmark our method.

\section{B. Template Construction}

For a given protein sequence target, PSI-BLAST [16] is used to search against the NR (Non-Redundant) database with $\mathrm{E}$-value $=0.001$ and at most 3 iterations to generate the PSSM (Position Specific Scoring Matrix) data. Then, the PSSM is used to search against the Protein Data Bank (PDB) [17] for alignments with $E$-value $=10.0$. If known structures are available in PDB, their 8-state assignments are determined by the DSSP program and then a structural template is built for the correspondent residue positions.

\section{Encoding and Nural Network Model}

We use a window size of 15 residues for input encodings. Each residue is represented with 20 values from the PSSM data, 1 extra input to indicate if the residue window overlaps C- or N-terminal, 1 value for degree of similarity, and 8 values for structural information from template or context-based secondary structure scores. For a residue with available structural information, the corresponding secondary structure state is set to 1 while the other states are set to 0 . At the same time, the degree of similarity is set for the sequence similarity. On the other hand, if the structural information for a resi- 
due is not available, the degree of similarity is set to 0 and the context-based scores are incorporated instead. The context-based scores are heuristic statistics to specify the favorability of a residue adopting a certain secondary structure in its amino acid context. Detailed description of generating context-based scores can be found in [18].

We incorporate two phases of standard feed-forward neural network training. We use 7-fold cross validation on the training of protein sets. Q8 and SOV8 (Segment overlap [19]) scores are used to measure the prediction qualities.

\section{RESULTS AND DiscUSSIONS}

Upon the selection of the best alignment with similarity $<95 \%$ for all chains in Cull5547, an overall of $78.85 \%$ Q8 accuracy and $80.10 \%$ SOV8 accuracy are achieved in 7-fold cross validation. Table 1 compares the accuracy of using predictions with and without templates on the benchmarks. Clearly, when homology structural information is available, the prediction accuracy is significantly improved.

TABLE 1. COMPARISON BETWEEN 8-STATE PREDICTIONS WITH AND WITHOUT TEMPLATE ON CB513, CASP9, MANESH215, AND CARUGO338

\begin{tabular}{|r|l|c|c|c|c|}
\cline { 2 - 6 } \multicolumn{2}{c|}{} & CB513 & CASP9 & Manesh215 & Carugo338 \\
\hline \multirow{2}{*}{$\mathbf{Q}_{\mathbf{8}}$} & No Template & 67.22 & 71.54 & 69.71 & 68.44 \\
\cline { 2 - 6 } & With Template & 79.39 & 76.36 & 81.10 & 80.39 \\
\hline \multirow{2}{*}{$\mathbf{S O V}_{\mathbf{8}}$} & No Template & 67.66 & 73.47 & 70.79 & 69.50 \\
\cline { 2 - 6 } & With Template & 80.64 & 78.15 & 82.99 & 81.95 \\
\hline
\end{tabular}

\section{CONCLUSIONS}

We describe a template-based approach to enhance 8state secondary structure prediction accuracy in this paper. Our computational results show that the templates can help improve the prediction accuracy. Overall, $78.85 \% \mathrm{Q}_{8}$ and $80.10 \% \mathrm{SOV}_{8}$ accuracies are achieved in 7-fold cross validation. The effectiveness of using templates has been demonstrated on the benchmarks. A webserver (C8-Scorpion) implementing 8-state secondary structure prediction is available at http://hpcr.cs.odu.edu/c8scorpion. The integration of template-based prediction into the C8-Scorpion webserver is currently under development.

\section{ACHKNOWLEDGEMENTS}

This work is partially supported by NSF grant 1066471 and ODU 2013 Multidisciplinary Seed grant.

\section{REFERENCES}

[1] B. Rost, "Review: Protein secondary structure prediction continues to rise," Journal of Structural Biology, vol. 134, pp. 204-218, May-Jun 2001.

[2] D. T. Jones, "Protein secondary structure prediction based on position-specific scoring matrices," Journal of Molecular Biology, vol. 292, pp. 195-202, Sep 171999.
[3] B. Rost and C. Sander, "Combining evolutionary information and neural networks to predict protein secondary structure," ProteinsStructure Function and Bioinformatics, vol. 19, pp. 55-72, May 1994.

[4] G. Pollastri and A. McLysaght, "Porter: a new, accurate server for protein secondary structure prediction," Bioinformatics, vol. 21, pp. 1719-1720, Apr 152005.

[5] C. Cole, J. D. Barber, and G. J. Barton, "The Jpred 3 secondary structure prediction server," Nucleic Acids Research, vol. 36, pp. W197-W201, Jul 2008.

[6] G. Pollastri, D. Przybylski, B. Rost, and P. Baldi, "Improving the prediction of protein secondary structure in three and eight classes using recurrent neural networks and profiles," Proteins-Structure Function and Genetics, vol. 47, pp. 228-235, May 12002.

[7] B. Petersen, T. N. Petersen, P. Andersen, M. Nielsen, and C. Lundegaard, "A generic method for assignment of reliability scores applied to solvent accessibility predictions," Bmc Structural Biology, vol. 9, Jul 312009.

[8] W. Kabsch and C. Sander, "Dictionary of protein secondary structure: pattern recognition of hydrogen-bonded and geometrical features," Biopolymers, vol. 22, pp. 2577-637, Dec 1983.

[9] Z. Wang, F. Zhao, J. Peng, and J. Xu, "Protein 8-class secondary structure prediction using conditional neural fields," Proteomics, vol. 11, pp. 3786-92, Oct 2011.

[10] G. Pollastri, A. J. M. Martin, C. Mooney, and A. Vullo, "Accurate prediction of protein secondary structure and solvent accessibility by consensus combiners of sequence and structure information," Bmc Bioinformatics, vol. 8, Jun 142007.

[11] J. Roland L. Dunbrack. A protein sequence culling server. Available: http://dunbrack.fccc.edu/Guoli/pisces_download.php

[12] J. A. Cuff and G. J. Barton, "Application of multiple sequence alignment profiles to improve protein secondary structure prediction," Proteins-Structure Function and Genetics, vol. 40, pp. 502-511, Aug 152000.

[13] S. Ahmad, M. M. Gromiha, and A. Sarai, "Real value prediction of solvent accessibility from amino acid sequence," Proteins-Structure Function and Genetics, vol. 50, pp. 629-635, Mar 12003.

[14] O. Carugo, "Predicting residue solvent accessibility from protein sequence by considering the sequence environment," Protein Engineering, vol. 13, pp. 607-609, Sep 2000.

[15] L. N. Kinch, S. Shi, H. Cheng, Q. Cong, J. M. Pei, V. Mariani, T. Schwede, and N. V. Grishin, "CASP9 target classification," ProteinsStructure Function and Bioinformatics, vol. 79, pp. 21-36, 2011.

[16] S. F. Altschul, T. L. Madden, A. A. Schaffer, J. Zhang, Z. Zhang, W. Miller, and D. J. Lipman, "Gapped BLAST and PSI-BLAST: a new generation of protein database search programs," Nucleic Acids Research, vol. 25, pp. 3389-402, Sep 11997.

[17] H. M. Berman, J. Westbrook, Z. Feng, G. Gilliland, T. N. Bhat, H. Weissig, I. N. Shindyalov, and P. E. Bourne, "The Protein Data Bank," Nucleic Acids Research, vol. 28, pp. 235-42, Jan 12000.

[18] Y. Li, H. Liu, I. Rata, and E. Jakobsson, "Building a KnowledgeBased Statistical Potential by Capturing High-Order Inter-residue Interactions and its Applications in Protein Secondary Structure Assessment," J Chem Inf Model, vol. 53, pp. 500-8, Feb 252013.

[19] A. Zemla, C. Venclovas, K. Fidelis, and B. Rost, "A modified definition of Sov, a segment-based measure for protein secondary structure prediction assessment," Proteins-Structure Function and Genetics, vol. 34, pp. 220-3, Feb 11999. 